\title{
Design of Drawer-type Random Drawing Device Based on TRIZ Theory
}

\author{
Chi Ningjun ${ }^{1}$, Yu Tongan ${ }^{2}$ \\ ${ }^{1}$ Art College, Xi'an University of Science and Technology, Shaanxi, 71000, China \\ ${ }^{2}$ Art College, Xi'an University of Science and Technology, Shaanxi, 71000, China
}

\begin{abstract}
Through the redesign of the traditional drawing cylinder, this paper explores the innovative design of the integration of traditional culture and modern technology in the experience tourism environment. Based on TRIZ theory, this paper determines the existing problems, applies the technology contradiction matrix to obtain the corresponding invention principle, constructs the substance-field model to deduce the best contradiction solution, and finally realizes the drawer-type drawing device with the function of distributing random and unique fortune sticks. The drawer-type random drawing device realizes the integration of traditional culture and modern technology, and improves the tourism experience.
\end{abstract}

\section{Introduction}

In the era of experience economy, tourists pay more and more attention to obtaining specific experience in the process of tourism which meets their psychological needs and interest preferences. In this trend, more and more experiential and emotional experience tourism has emerged and flourished. Providing personalized, experiential and emotional tourism can give tourists an extraordinary tourism experience [1]. Based on TRIZ theory, this paper designs a new type of drawing device according to the existing problems of draw products, so as to create a better experience scene and enhance the tourists' sense of experience.

\section{Overview of TRIZ theory}

TRIZ theory was founded in 1946 by G.S. Altshuller, the inventor of the former Soviet Union, and is now widely used in the field of innovative design of product. The theory follows the objective law, starts with product analysis, gradually goes deeper to seek for the innovative principles of conflict and problem solving, and finally forms a feasible design scheme, which realizes as many functions as possible with as few resources [2]. The problem solving mode and process are shown in Figure 1.

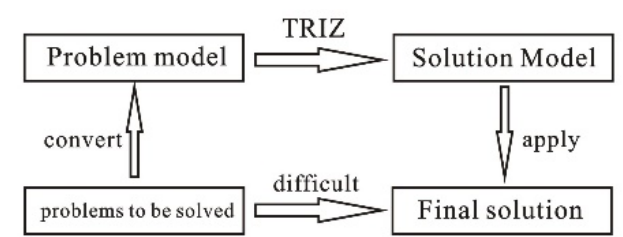

Figure 1 Problem solving mode and process based on TRIZ theory

\section{Cases of existing product design}

\subsection{Function analysis}

1406646868@qq.com 221806634805@qq.com
The purpose of function analysis is to analyze problems from the perspective of completing functions, including whether each function is necessary. Significant breakthroughs in design and significant reductions in cost or complexity are often the result of function analysis [3]. The traditional drawing device only has the function of placing the fortune sticks. 
Tourists need to shake the stick cylinder to wait for the first one to fall out or randomly select a stick from it. After opening the app for draw, users can click the buttons in the app to pray and draw fortune sticks online.

\subsection{Problem description and analysis}

(1) Problem description. The traditional drawing cylinder structure and drawing method are too simple and easy to make mistakes. Mobile phone can improve the efficiency of draw, but users have a poor experience of doing it through the screen.

(2) Problem analysis. We should put people at the center of service, deeply understand people's needs, and complete valuable service experience design [4]. From the user feedback and the analysis of product functions, use problems and market conditions, it can be found that many scenic spots have not improved the traditional drawing cylinder structure and drawing method, and the users experience of directly randomly drawing from the stick cylinder is not strong, and they still need to draw again when there are multiple sticks shaken out, which wastes time. Due to the lack of physical interaction in mobile phone operation and the loss of traditional cultural connotation in the pray-anddraw process, online drawing is unable to respond well to the emotional demands of the prayer. The tourist's tourism experience is finally reflected in the product function and modeling. The function problems of the traditional drawing cylinder are imperfect function, poor experience and low efficiency; the modeling problems are weak operation semantics and too simple modeling. The function problem of mobile app is the poor user experience; the modeling problem is the poor form of draw.

\subsection{The establishment and solution of contradiction}

According to the existing product function and modeling problems to sum up the contradiction [5]: contradiction 1 is the impact of technology on the traditional drawing cylinder modeling. Without modern technology, it can't solve the negative psychological emotions of tourists; while if technology is involved too much in the modeling of drawing cylinder, it will affect the sense of ceremony of praying and drawing just like the mobile app. This problem can be transformed into 39 general engineering parameters: the technical feature to be improved is No. 33 (operability), and the technical feature of deterioration is No. 34 (maintainability). Now they are substituted into the TRIZ contradiction matrix table, as shown in Table 1. The invention principles obtained are: Article 35 (performance conversion method), Article 1 (segmentation method), Article 11 (principle of preemergency measures) and Article 9 (principle of prereaction).

Contradiction 2 is the impact of the time spent by tourists on drawing cylinder on the actual experience. Tourists tend to spend more time drawing fortune sticks. However, the long waiting time will reduce the enthusiasm of tourists. The problem can be transformed into 39 general engineering parameters: the technical feature to be improved is No. 25 (time loss), and the corresponding technical feature of deterioration is No. 12 (modeling). They are substituted into the TRIZ contradiction matrix table, as shown in Table 1. The invention principles obtained are as follows: Article 4 (asymmetric principle), Article 10 (pretreatment principle), Article 34 (principle of discarding or regenerating parts) and Article 17 (principle of conversion to new dimension).

Table 1 Technology contradiction matrix of TRIZ method

\begin{tabular}{ccc}
\hline Technical feature to be & \multicolumn{2}{c}{ Technical feature of deterioration } \\
\cline { 2 - 3 } improved & 12.Modeling & 34. Maintainability \\
\hline 25.Time loss & 4.10 .34 .17 & 32.1 .10 \\
33. Operability & $15,34,29,28$ & $35,1,11,9$ \\
\hline
\end{tabular}

There is also contradiction 3 of drawing cylinder: the contradiction in the internal space of the drawing cylinder. The staff need to put a large number of fortune sticks in the cylinder, that is, the input quantity required is large; while when the tourists draw fortune sticks, only one stick needs to be taken out each time, so we need to find a stable and limited output of the drawing cylinder. Therefore, it is necessary to separate the two sides of the contradiction under different conditions through the method of conditional separation to reduce the difficulty of solving the problem [6]. The principles of invention corresponding to the principle of conditional separation are as follows:
Article 1 (division method), Article 5 (combination principle), Article 6 (multi-use of one thing), Article 7 (nesting principle), Article 8 (method of skillfully taking heavy objects), Article 13 (reverse thinking method), Article 14 (spherizing method), Article 22 (method of changing harm into benefit), Article 23 (feedback principle), Article 25 (self-service method), Article 27 (substitution method), Article 33 (assimilation method) and Article 35 (performance transformation method).

See Table 2 for the list of the above principles ordered by the frequency of occurrence from high to low. There are 23 principles in Table 2, among which 
Article 1 (segmentation method) has a frequency of three, Article 10 (pretreatment principle) and Article 35 (performance conversion method) have a frequency of two, and the other 20 principles have a frequency of one. Therefore, the segmentation method, pretreatment principle and performance conversion method are used to design the structure and function of the drawing device.

Table 2 Combination and sorting of invention principles

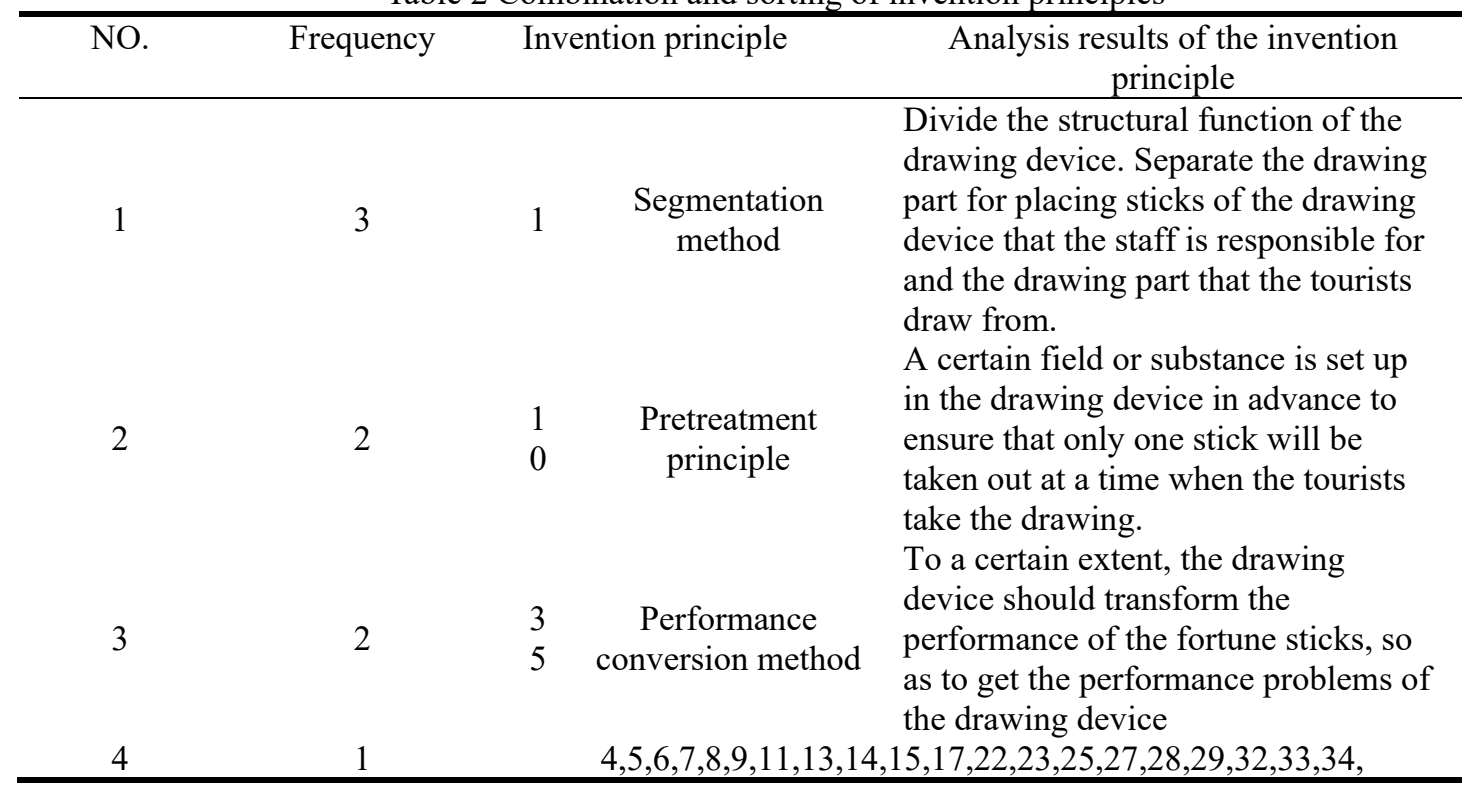

\section{Substance-field analysis}

The substance-field model can be used to analyze model problem related to existing technologies [7]. The substance-field model of the drawing device is shown in Figure 2, where $\mathrm{F}$ is the force field, $\mathrm{S} 2$ is the drawing cylinder, S1.1 is the fortune sticks put in by the staff, S1.2 is the only stick drawn by the tourist every time, the straight arrow represents the expected effect or influence, and the curve arrow represents the harmful effect or influence. In this model, the fortune sticks to be taken out can be placed in the drawing device, which is the expected effect. However, because there are too many sticks in the drawing device, it will affect the effect of tourists taking only one stick at a time, so it is the harmful effect. Through the analysis of the substance-field model, it can be seen that substance similar to "door" needs to be added to restrict the movement of other fortune sticks: S3 (angle block) and F2 (a certain field) of another field to ensure that after taking out one stick, the subsequent sticks will be blocked by "door", so as to achieve the goal that tourists can only take out one stick at a time.

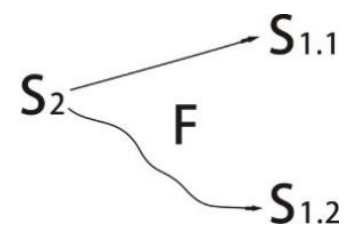

Figure 2 Substance-field model of drawing device

In addition to the new substance, the traditional drawing cylinder still needs a force field to ensure that after changing the modeling of the cylinder, this force field can cooperate with S3 (angle block) to solve the problem. Through the analysis of resource type, acquisition difficulty, quantity and availability [8], there are atmospheric pressure, geomagnetic field and gravity field which comply with the request. If air pressure and magnetic field are involved in the drawing system, the air tightness and magnetism of the system should be considered, which will complicate the drawing device. The gravity field not only is readymade and free, but also makes it easy to restrict the movement of sticks by adjusting the shape of the sticks through gravity field and angle block, such as changing into cylinder, so as to realize the automatic control of the drawing device to the fortune sticks. 


\section{Structure design}

\subsection{Modeling design}

Considering that the addition of technological innovation should not affect the original traditional cultural ideas of the automatic stick-out drawing device, the modeling learns from the characteristics of lacquer wood furniture in Ming and Qing dynasties [9], such as the Buddha's Ark in the sixth year of Chongzhen, the huanghuali wood bright shelf cabinet with veneer carved with dragon pattern and the huanghuali wood long narrow table with round legs. Based on the analysis of the carving style and overall atmosphere of the above furniture, combined with the functions required by the drawing device, the relevant features are extracted to determine the final drawertype random drawing device modeling. The axonometric drawing and front view are shown in Figure 3. The overall modeling of the device is classic and elegant, and the appearance style can also be adjusted according to the characteristics of the scenic spot.
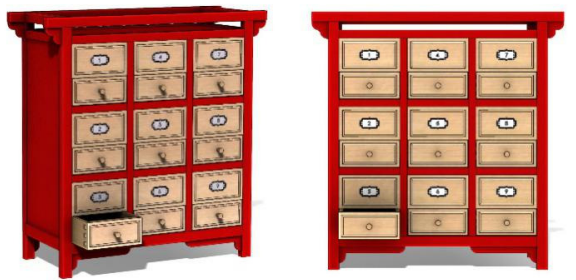

Figure 3 Axonometric drawing and front view

\subsection{Internal structure design}

Figure 4 shows the internal structure of the automatic stick-out drawing device designed according to TRIZ theory. The cabinet body of the drawer-type random drawing device is provided with hidden layers and a drawer box. At the same time, each group of hidden layers corresponds to the drawer box one by one, as the number shown in Figure 3. There are nine groups of draw boxes in total, and different fortune sticks can be put into different drawer boxes. And the drawer box is provided with a pull ring, which is convenient for tourists to draw at the same time. In each group, the hidden layer is connected with the corresponding drawer box through the stick filter system. The back of

the hidden layer is provided with a back box cover, and the bottom of the hidden layer is a rectangular trapezoid hopper. There is an outlet on the side of the hopper which is able to hold the only sticks.

The most important mechanism in the stick filter system is the angle block. The angle block is an Lshaped metal groove, which is fixedly connected with both sides of the rectangular trapezoid hopper through a metal fixing plate and two fixing nails. The bottom of the angle block and the metal fixing plate goes deep into the drawer box. The angle block itself is composed of a rotating shaft and left and right sides, and the rotating shaft is movably connected with the metal fixing plate. The angle block serves as an important jointing element, with one side in contact with the side of the outlet and the other side connected to the drawer box below by a spring.
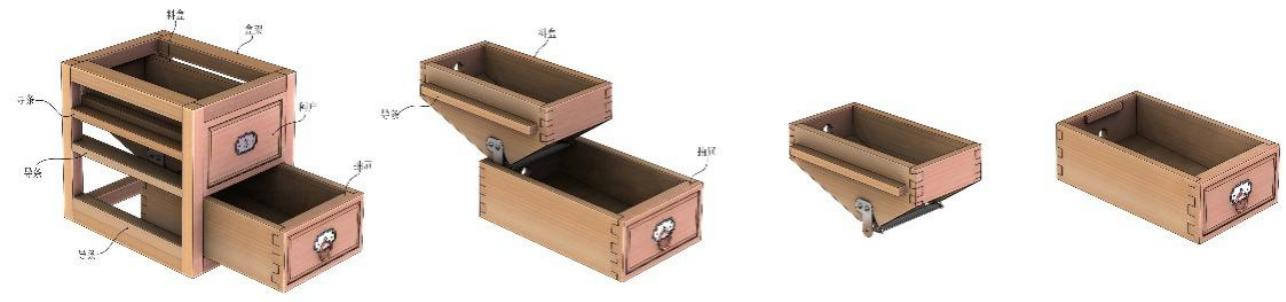

Figure 4 Internal structure of the device, material box and drawing box

\subsection{Operating principle}

The cabinet body contains nine groups of hidden layers and drawer boxes, which can be used to receive a variety of different types of sticks. The staff can put in the fortune sticks after opening the back box cover. The stick rolls into the bottom of the hidden layer.
When the tourists pull the pull ring to pull out the drawer box, the cushion block behind the drawer box pushes the upper edge of the back plate of the drawer box, forcing the angle block to rotate around the rotating shaft. When the drawer box and cushion block can't be drawn out more due to the limitation of metal fixing plates on both sides, the front side of the angle block is in a downhill shape. At this time, the stick will 
roll into the drawer box from the front side of the angle block due to the influence of its own gravity. The internal situation of drawer box in the process of drawing fortune sticks is shown in Figure 5. Only one stick in the hidden layer can fall into the angle block, and the rest will be blocked by the side edge of the angle block, so that they cannot fall into the drawer box. Moreover, the upper part of the angle block after rotating can prevent other sticks from rolling off, and the lower part can also guide the only stick to roll out.
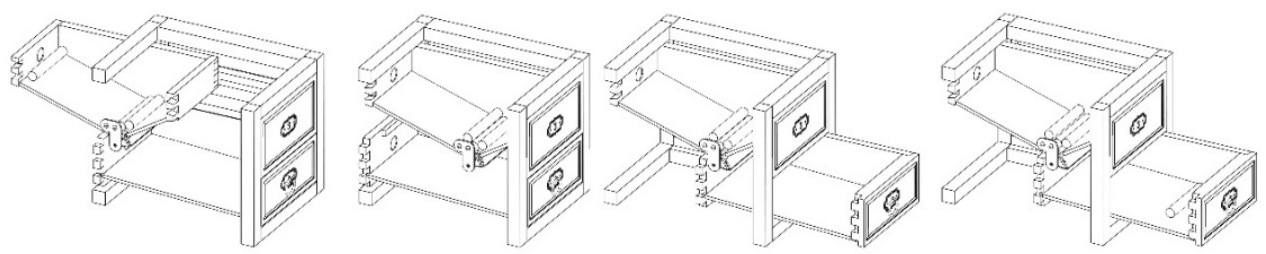

Figure 5 Process of opening the back box cover, placing the fortune sticks, opening the drawing box and the falling of the stick

\section{Experiencing draw fortune sticks}

Tourists can experience drawer-type random drawing device by scanning code using mobile phone or under the guidance of staff. According to the actual needs, the staff of the scenic spot will set nine groups of draw boxes with fortune sticks for "mark for task" in the scenic spot. When a tourist draws a "mark for task" type fortune stick, they need to take a group photo to mark at the designated location of the scenic spot within the specified time according to the instructions on the fortune stick, and receive gifts after completing the task. The whole drawing process is similar to the black box. Tourists can experience the wonderful feeling of the fortune stick rolling out when pulling the drawer. After the fortune stick is drawn, tourists can experience the pleasure of completing the task according to the instructions on the stick. Finally, tourists can also keep these fortune sticks as souvenirs of their own experience in the scenic area.

\section{Conclusion}

In the era of experience tourism, aiming at the problems in the use of traditional products in scenic spots, this paper innovatively applies TRIZ theory to drawing products. We combine traditional products with appropriate technological innovation, make product design as a positive cultural behavior, and give humanistic value beyond product functionality [10]. While inheriting the traditional culture, it expands the relevance between technology and culture, and realizes the purpose of improving the tourist experience.

\section{References}

1. Tian Qin-si, Wang Ye, Zhu-zhu, He Ping. (2017) Development Strategy of Experiential
Tourism in Famous Historical and Cultural Villages [J] .Agricultural Economy, 06: 54-56.

2. Zhang Wei-guo, Zhang Guo-quan, He Hai, et al. (2005) Using TRIZ theory to solve the problem of innovative design of complex electromechanical products $[\mathrm{J}]$. Mechanical Design and Research, 21 (3): 15-18.

3. Tan Run-hua, WANG Qing-yu, YUAN Caiyun, et al.(2001) Theory for Solving of Solving the Inventive Problems (TRIZ): the Process Tools and Developing Trends of TRIZ [J]. Mechanical Design,(7): 7-11.

4. Zhao Fang.(2018) On the Core Value of Service Design Research [J] .Grand View of Fine Arts, 10: 126-127.

5. Dai Qing-hui, MA Xi-kun. (2012) A New Method for Determining Ideality Based on TRIZ Contradiction Matrix [J] .Mechanical Design \& Research, 28 (6): 11-13.

6. Ai Xian-feng, Hu Kang, Zhou Hong-yu, et al. (2015) Research on Industrial Design Innovation Process Based on TRIZ [J]. Mechanical Design, 32 (11): 105-109.

7. Tan Run-hua,ZHANG Huan-gao. (2014) Interactive Training Model of TRIZ for Mechanical Engineers in China [J].Chinese Journal of Mechanical Engineering ,27(02):240-248.

8. Sun Zhi-xue. (2018) Improved Design of Household Refrigerator Drawers Based on TRIZ Theory [J].Packaging Engineering, 39(14):18-21.

9. Li Ji-xia. (2019) Research on the Idea of Creation in Ming Dynasty Furniture Design [J]. Packaging Engineering, 39(16):214-219.

10. Fang Dao-Rong.(2012) Research on the Innovative Design of Handicrafts in the Background of "Qiao Niang Studio" [D]. Tsinghua University. 